\title{
LAS EXPRESIONES AUTONÓMICAS DEL CONO SUR: JAGUARIBE, PUIG TOMASSINI, LINCK Y O'DONNELL ${ }^{(*)}$
}

\author{
THE AUTONOMOUS EXPRESSIONS OF SOUTH CONER: JAGUARIBE, \\ PUIG, TOMASSINI, LINCK AND O'DONNELL
}

Alejandro Simonoff $f^{*}$

Universidad de La Plata, La Plata (BA), Argentina

Resumen: Las diversas expresiones de la teoría de la autonomía se han mostrado a lo largo del tiempo, como un fenómeno a la vez único y diverso al mismo tiempo. Para observar la complejidad de este fenómeno analizaremos las propuestas teóricas de Linck y O’Donnell, Jaguaribe, Puig y Tomasini. Ellas son únicas por el tiempo y espacio en el que fueron pensadas, pero además poseen aspectos en los que difieren. Para explicarlo debemos analizar sus fuentes (el Segundo Debate paradigmático y las teorías del Centro-Periferia) que ayudan a comprender sus diferencias.

Palabras Claves: CEPAL; Teoría de la Dependencia; Autonomía.

Abstract: The various theories of autonomy have been analyzed over time, as a phenomenon unique and different at the same time. To witness the complexity of this phenomenon we will discuss the theoretical proposals of Linck and O'Donnell, Jaguaribe, Puig and Tomasini. They are unique in time and space in which they were proposed, but also possess aspects that differ from others. To explain this singularity we will analyze their sources (the Second Debate paradigm and theories of center-periphery).

Keywords: ECLAC; Dependency Theory; Autonomy.

(*) El autor es muy grato por la la lectura y comentario de la Licenciada Victoria Zapata.

$(* *)$ Doctor, Profesor del Instituto de Relaciones Internacionales de la Universidad Nacional de La Plata (IRI/UNLP). E-mail: <asimonoff2010@gmail.com>. Recibido en: 22.09.2014, aceptado en: 05.11.2014. 


\section{INTRODUCCIÓN}

Las diversas expresiones de la teoría de la autonomía se han mostrado a lo largo del tiempo, como un fenómeno a la vez único y diverso al mismo tiempo.

Para observar la complejidad de este fenómeno analizaremos las propuestas teóricas de Linck y O'Donnell, Jaguaribe, Puig y Tomasini. Ellas son únicas por el tiempo y espacio en el que fueron pensadas, entre fines de los sesenta y principios de los ochenta en el Cono Sur de América. Pero los autonomistas, a pesar de compartir diversos puntos de sus propuestas, poseen aspectos en los que difieren.

Creemos que para explicar esto debemos analizar sus fuentes y si cómo ellas cómo se van diferenciando cada una de las propuestas.

\section{LA UNIDAD}

La aparición de estas contribuciones latinoamericanas se sustentan en dos fuentes: el Segundo Debate paradigmático y las teorías del Centro-Periferia. A continuación veremos cómo es relacionan.

\subsection{EL SEGUNDO DEBATE PARADIGMÁTICO Y SU INCIDENCIA EN LOS ESTUDIOS LATINOAMERICANOS}

Este debate se produjo durante los años cincuenta y sesenta. Esta discusión entre los llamados tradicionalistas (realistas) y los cientificistas (behavoristas), se inició ante la crítica de estos últimos a los primeros, quienes entendían que sus estudios se basaban "en intuiciones no sustentadas por una labor científica de verificación empírica". En cambio los primeros sostuvieron que los segundos construyeron "hipótesis banales o que sus descubrimientos eran irrelevantes" (TOMASSINI, 1980, p. 551).

Este debate tiene múltiples aspectos, desde cómo evoluciona el conocimiento científico, cuáles son los niveles de análisis, si se pueden elaborar teorías, etc.

Tomaremos un aspecto de éste que nos parece relevante: la cuestión referida a los niveles de análisis. Estas nuevas claves sirven para explicar el funcionamiento del conjunto de la sociedad internacional y son denominadas teorías de alcance medio. Las teorías parciales se concentran en una región o fenómeno concreto (teorías de integración), o ambas, o a un determinado tipo de actores (teoría de decisión).

Esta cuestión referida a los actores que intervienen en la decisión, es un punto central, ya que ataca el corazón de la teoría realista que entiende que el Estado es un actor único y racional, y lo desarticula en varios actores que inciden en la acción de éste.

A pesar que desde el Programa de Estudios Comparados sobre Relaciones Internacionales de América Latina (RIAL), este debate pasó inadvertido (TOMASSINI, 1980, p. 546), su consecuencias no, ya que sin él no se puede comprender la aparición del Autonomismo.

Pero volviendo al rol de los actores en el escenario internacional, si tomamos a Juan Carlos Puig observamos la disputa entre elites (diferenciadas por diversos modelos 
de inserción del Estado periférico), aunque utiliza a autores realistas (Raymond Aron, Morton Kaplan y Klaus Knor) como fuentes, no puede decirse que sea un realista clásico.

Para tomar un ejemplo, de esto para nuestro autor el Interés Nacional "tiene que ver no sólo con la protección física de la nación (territorio y población), sino también con la del "estilo de vida" (PUIG, 1984, p. 49) Esta definición es de una innegable factura aroniana (ARON, 1962, p. 16), más que morgenthiana.

En estos términos identificamos la obra puigiana con la tradición realismo, como dice Dallanegra, es de una cosmovisión realista de fines, no de medios (PEDRAZA, 2009, p. 129), o como lo hace Raúl Bernal Meza que la entiende como "una estrategia "posible" de integración y autonomía en el sistema internacional" (MEZA, 2013, p. 50).

No negamos la inscripción de éste en el realismo, lo que impugnamos son aquellas perspectivas que lo identifican con el realismo morgentheano, como una perspectiva vetusta de las relaciones internacionales frente a la interdependencia compleja y el institucionalismo neoliberal que influyeron en las escuelas de Carlos Escudé y Roberto Russell (CORIGLIANO, 2009) ${ }^{(1)}$.

Por ello creemos acertado ver el origen de este paradigma como una convergencia de varias percepciones, lo que Arlene Tickner denominó como "condiciones de hibridación", "caracterizadapor la incorporación de supuestos y conceptos derivados de la teoría de la dependencia, el realismo clásico y la interdependencia..." (TICKNER, 2002, p. 19).

\subsection{LAS TEORÍAS CENTRO-PERIFERIA}

En segundo lugar, los aportes de las teorías de Prebisch y de la Teoría de la Dependencia, son el otro núcleo de fuentes. Estas plantearon que el subdesarrollo no culmina en el desarrollo - como defiende la teoría de la modernización -, sino que era parte fundamental e inseparable del capitalismo avanzado, es decir, ambos son co-constitutivos. Es una parte funcional del desarrollo del centro dentro de la lógica de la división internacional del trabajo.

\subsubsection{LA CEPAL}

La Comisión Económica para América Latina identifica la causa de la brecha entre Centro y Periferia, como el producto del deterioro de los términos del intercambio. En el marco de la especialización productiva, pero a diferencia de las teorías clásicas, los precios de los productos de alto valor agregado fueron mucho más elásticos que los de los precios agrícolas, y ello contribuyó al aumento de la brecha entre países desarrollados y subdesarrollados.

La Teoría de Prebisch dio "un fundamento de análisis económico y un basamento empírico, así como apoyo institucional, a la búsqueda de bases autónomas de desarrollo" (SAN-

(1) La primera identificación de este tipo la encontramos en Van Klaveren, quien ubica indirectamente a Puig en el campo de los análisis del poder, al señalar que su Revista Argentina de Relaciones Internacionales esta en los parámetros del realismo y de la geopolítica (KLAVEREN,1984, 24-26). 
TOS, 2003, p. 62). El camino a seguir para salir de este estadio era la industrialización que se lograría a través de tres medios: la superación de la sustitución de importaciones, la integración regional y la defensa de los precios internacionales de las materas primas.

La sustitución de importaciones es una primera etapa de este proceso, aunque Prebisch reconoce que no era el modo más apropiado, y que a partir de allí habría que superarla a través de dos formas del proceso de acumulación: con la utilización de las divisas resultantes del comercio exterior y la incorporación de capitales extranjeros en la medida que no afectasen la capacidad decisoria de la nación y con gran control estatal, que permitirían un grado mayor de industrialización hacia la producción de bienes intermedios, de capital y de consumo durable.

El impulso de la integración regional se sustenta en favorecer los intercambios recíprocos, a través de la formación de un mercado común latinoamericano que promueva la exportación de manufacturas propias.

La creación de instrumentos para defender los precios de los productos primarios se realiza a través de dos vías: la defensa individual de ellos para poner fin a estas distorsiones y la colectiva, impulsando acuerdos de estabilización e impugnación de las teorías neoclásicas que sostienen esta situación de inequidad.

Aunque estos instrumentos sufrieron modificaciones a partir del derrotero de esta corriente desde los años setenta, en esa década propone "evitar el endeudamiento excesivo" y que se debía "buscar la complementariedad entre la industrialización sustitutiva y la expansión de las exportaciones industriales". En el decenio siguiente, y producto del crecimiento y crisis de las deudas externas latinoamericanas se plantea como medidas "la expansión exportadora combinada al crecimiento, o sea, al "ajuste con crecimiento" (BIELSCHOWSKY, 2011, p. 14).

En este marco teórico no hay discusión del paradigma capitalista, sino la presentación de una respuesta estructuralista que difiere de la versión liberal. Al respecto, Puig puntualiza que este programa no tiene nada "que signifique el cuestionamiento al propio régimen (capitalista) que es el generador de la injusticia" (PUIG, 1984, p. 15).

Pero del mismo modo, este último aspecto fue el que generó críticas dentro de la propia CEPAL, ya que el propio Prebisch reconocía que podían eliminarse las causas del subdesarrollo y sin embargo éste podía persistir (IÑIGUEZ, 2003, p. 188). En la crítica de las potencialidades reales de este modelo de desarrollo hacia adentro se encuentran los fundamentos de la teoría de la dependencia, que sostiene que el subdesarrollo es un producto histórico del capitalismo y del establecimiento de la división internacional del trabajo que moldea la economía latinoamericana a las exigencias del mercado mundial ${ }^{(2)}$.

Para Theotonio dos Santos, la CEPAL "representó el auge de la ideología nacional-desarrollista en América Latina y el Tercer Mundo" pero había "confiado demasiado en el papel de la industrialización para garantizar la modernización económica y la creación de

(2) Además de estas críticas al pensamiento cepalino, algunos autores señalan la existencia de otra fuente más en el origen de la Teoría de la Dependencia, el debate entre el marxismo clásico y el neomarxismo entre Paul Baran y Paul Sweezy (SANTOS, 2003: 25). 
centros nacionales de decisión económica o de acumulación capitalista” (SANTOS, 2003, p. 106-107).

\subsubsection{TEORÍA DE LA DEPENDENCIA}

Como dijimos anteriormente y de acuerdo con la CEPAL, el subdesarrollo es una característica fundamental en el sistema capitalista. Sin embargo, la Teoría de la Dependencia analiza la dependencia más allá de las cuestiones económicas, en sus planos políticos, ideológicos y culturales.

$\mathrm{Su}$ originalidad estuvo "en mostrar la articulación entre las clases sociales y, especialmente, entre las fracciones de las clases dominantes y también el papel del Estado en el proceso de internacionalización del capital” (NOVOA, 1979, p. 77).

Los principales aspectos de la Teoría de la Dependencia son, según Santos (2003, p. 23):

I. el desarrollo está estrechamente ligado a la expansión industrial en los países desarrollados;

II. el desarrollo es parte de un proceso universal;

III. el desarrollo no puede ser considerado como la primera condición de un proceso evolutivo;

IV. la Dependencia no es un fenómeno únicamente externo, sino que también se manifiesta internamente en los países en los ámbitos social, económico y político.

Existen tres formas distintas de la Teoría de la Dependencia, una versión marxista, o neomarxista en realidad, una corriente estructuralista y una versión crítica dentro del pensamiento de CEPAL (KAY, 1998, p. 103).

La primera de ellas está representada por André Gunder Frank, de matriz marxista, y su teoría del subdesarrollo. Su aporte estuvo en "vincular la relación centro-periferia al interior de los Estados, explicando cómo los gobiernos/elites fueron funcionales a la extranjerización de las tierras, del comercio, etc." (RASCOVAN, 2013, p. 29). Sus críticos plantean que la relación desarrollo-subdesarrollo es inherente al capitalismo y por ello se impediría el desarrollo para el Tercer Mundo. Esto es rechazado por los miembros de esta corriente aunque sostienen que el "desarrollo capitalista" se daba plenamente, aunque de forma dependiente y desigual (PRADO, 2013, p. 113). Aquí podríamos ubicar además a Theotonio dos Santos, Ruy Marini y Samir Amin desde el sur, la teoría del Sistema Mundo de Emanuel Wallerstein y las lecturas de Giovanni Arrighi.

Para la segunda corriente, concentrada en la figura de Fernando Henrique Cardoso, la dependencia es concebida como el resultado de relaciones internas y externas de clases, aunque reconociendo la existencia de un desarrollo capitalista asociado.

Las burguesías nacionales de la periferia sirven a los intereses de la propagación del capital extranjero, lo que genera un sistema económico dependiente y subordinado a éste y que el aumento de sus ingresos relacionados con la exportación/importación no se traduce en el desarrollo del resto del tejido social. En 1980, Cardoso reajusta su 
pensamiento a una versión más tenue que propone un carácter cooperativo y no de ruptura con el centro para el desarrollo capitalista asociado. Y a partir de aquí este autor sostuvo que se hizo una lectura errónea de su trabajo con Enzo Faleto, Desarrollo y Dependencia en América latina, ya que no se entendió "el sentido que debamos a la noción de capitalismo asociado (entre el centro y la periferia) y enfatiza la idea de la dependencia como impedimento al desarrollo" (CARDOSO, 2011, p. 31). Este cambio se explica por dos razones principales:

I. la onda neoliberal internacional que fue transformando la economía global;

II. porque es un período de revalorización de la democracia como sistema político (reflejo de la decadencia soviética y de las dictaduras de América del Sur).

Finalmente, y como hemos adelantado en el apartado anterior, están también quienes proponen reformas al pensamiento de la CEPAL/Prebisch, como Osvaldo Sunkel y Celso Furtado, quienes hacen hincapié en las variables internas que mantienen el subdesarrollo. En el caso del trabajo del primero, Capitalismo transnacional y desintegración nacional en América Latina (1972, donde se plantea la alta convergencia entre los sectores integrados a la economía mundial, sean estos de los países centrales y periféricos, y la falta de complementariedad de los sectores no integrados en los extremos del sistema.

La Teoría de la Dependencia cree que el ciclo de desarrollo crea nuevos problemas, relacionados con el sector de la sociedad de capital externo y por lo tanto tienden a actuar en contra del interés nacional y en favor del interés reducido (este proceso crearía burguesías nacionales, dependientes y conservadoras). Por esta razón, la clave para romper el elemento de dependencia sería la movilización popular democrática con el fin de añadir al juego político de las masas, que fueron marginadas en la historia de América Latina. Sería una forma de contrarrestar la influencia política del capital extranjero en las franjas meridionales democracias frágiles.

A pesar de lo acertado de estos análisis, la propuesta de una salida por fuera del capitalismo se convertía en una opción descorazonadora y nihilista, como lo apuntó Juan Carlos Puig. "(...) no cabe duda de que tales asimetrías existen; de lo que se trata es de superarlas mediante maniobras estratégicas que se basen en un diagnóstico político acertado" (PUIG, 1984, p. 49).

Por ello, como apunta Bernal Meza:

(...) Puig supo escapar de un enfoque que por su visión cíclica de la historia que consideraba al conflicto como algo recurrente y suprahistórico, aparecía funcional a los intereses de aquellos Estados en posesión de un potencial de destrucción masiva, que fundamentaba la impermeabilidad intrabloques, para transitar nuevas perspectivas teóricas aptas para fundamentar estrategias autonomizantes. (MEZA, 1989, p. 237)

Puig supo tomar de las teorías cepalinas y dependentistas sus fortalezas, y también tomar en cuenta sus debilidades, pero como señala Bologna no podemos dejar de negar que ambas: 
(...) fueron un intento válido en medios académicos de buscar respuestas latinoamericanas al problema del subdesarrollo de la región y tratar de encontrar estrategias de superación de acuerdo a los márgenes de permisibilidad de la sociedad internacional, para el logro de desarrollos endógenos. (BOLOGNA, 1989, p. 251)

Estos planteos dieron las bases para que pensamientos como los de Helio Jaguaribe y Juan Carlos Puig tuvieran una base para ser enunciados.

Antes de pasar a las escuelas autonomistas propiamente dichas, veremos qué rol jugó la autonomía en esta corriente. Para ello resulta imprescindible el trabajo de Linck y O’Donnell Dependencia y Autonomía, allí se sostiene (1973, pp. 247-248) que para el logro de la primera era necesario:

I. la eliminación de las principales bases de operación del capitalismo dependiente;

II. cancelar las principales características internas del actual sistema de dominación;

III. una amplia participación popular en varios niveles;

IV. un incremento sustancial de la autonomía.

Muchos de estos elementos están presentes en los teorías de la autonomía de Jaguaribe y Puig: cambiar la situación de dependencia, remover los condicionantes internos, promover el acceso de las grandes mayorías al dominio del Estado; pero se diferencian de aquella, cuando la reduce a un medio, y no un fin como para ellos, un instrumento para la ruptura del sistema y de potencialidades ilimitadas.

\section{DIVERSIDADES AUTONÓMICAS}

Entonces para conceptualizar la autonomía vemos que en sus fuentes aparecen una primera bifurcación en dos versiones, una más radicalizada de ruptura y otra que no. Para dar cuenta de ello a continuación detallaremos como las tres expresiones elegidas ensayan un camino propio.

\subsection{LA AUTONOMÍA JAGUARIBERIANA}

Las limitaciones de la Teoría de la Dependencia permiten pensar que para salir de una "impotente marginación", la región podía avanzar en una propuesta, más modesta pero más efectiva, la autonomía periférica (KORNAT, 1981, p. 13).

Para comenzar con el análisis de la propuesta de Jaguaribe, nos parece interesante su concepto de construcción de política exterior. Ésta se construye a partir de: “(...) una ecuación de optimización, en el ámbito internacional, de los principales intereses de un país, teniendo en cuenta sus condiciones internas y externas y los medios de acción de que dispone." (JAGUARIBE, 1974, p. 104).

Para una adecuada ubicación del modelo de inserción internacional es necesaria: "La selección objetiva del modelo más adecuado para un país... (que) requerirá en consecuencia un análisis preliminar histórico-estructural del país en cuestión, a fin de poder determinar objetivamente sus principales rasgos y tendencias estructurales." (JAGUARIBE, 1973, p. 85). 
Si bien el autor brasileño centra sus análisis en claves explicativas "realistas”, por el fuerte énfasis en las capacidades de defensa, aunque a lo largo de su obra este aspecto se presenta más equilibrado con aportes de lo social, la economía y la cultura.

Para él (JAGUARIBE, 1979, p. 94), existen dos relaciones constitutivas del Orden Mundial de posguerra:

I. entre las superpotencias que determinan el sistema internacional por su oscilación entre fases de cooperación y de conflicto;

II. la centro-periferia existe en los todos sistemas imperiales: aunque pueden haber diferencias de acuerdo a cada periodo histórico.

Para determinar los requisitos para la existencia de autonomía, según este autor, son de dos órdenes: habilitatorios y ejecutorios.

En el pensamiento de Jaguaribe existen dos condiciones para analizar el funcionamiento de la autonomía: la viabilidad nacional y la permisividad internacional. El primero:

(...) el concepto de viabilidad nacional es esencialmente histórico y relativo. Lo determina fundamentalmente la relación existente entre los recursos humanos y naturales de que dispone una nación en un momento dado y el nivel de la tecnología existente en ese momento. (JAGUARIBE, 1973, p. 113)

Pero lo original de la propuesta de Jaguaribe está en el grado de cohesión interna, como un elemento básico de la viabilidad, ya que:

(...) Esta cohesión tiende a aumentar con el desarrollo global, en la medida en que este hace que aumenten, no solo el ingreso absoluto y per capita, sino también todos los otros indicadores económicos, sociales, culturales y políticos del desarrollo, con el correspondiente aumento de la participación en todos los planos (...) Las relaciones existentes entre las masas y las elites son susceptibles de convertirse en un conflicto que se auto-perpetúa y en una decreciente cooperación. El carácter dual de la sociedad divide en dos a la nación y socava la lealtad subjetiva de los sectores en conflicto hacia la sociedad nacional en conjunto. En esta forma, la vialidad nacional se ve minada por el desmembramiento interno. (JAGUARIBE, 1973, pp. 113-114)

El segundo, la permisividad internacional es:

(...) la medida en que, dada la situación geopolítica de un país y sus relaciones internacionales, este país dispone de condiciones para neutralizar el riesgo proveniente de terceros países dotados de suficiente capacidad para ejercer sobre él formas eficaces de coacción (...)

El orden ejecutorio está constituido por las alternativas que deben emprender los Estados Periféricos candidatos a la autonomía, ya sea porque:

(...) consiguen una satisfactoria autonomía técnico-empresarial en lo interno. b) compensatoriamente, logran disponer de condiciones favorables en sus relaciones con el centro imperial, en especial por identificaciones de orden étnico-cultural. (JAGUARIBE, 1973, p. 128) 
Las relaciones entre estas dos alternativas son las que conjugan la existencia de diversos niveles de auto-determinación: la primacía general, la primacía regional, la autonomía, y el nivel de dependencia. (JAGUARIBE, 1979, pp. 91-92).

La primacía general:

(...) se caracteriza por la combinación de la inexpugnabilidad del territorio propio con el ejercicio de una preponderancia mundial generalizada, que sólo está contenida en forma efectiva en las aéreas de inmediata hegemonía de una potencia dotada de primacía regional... (JAGUARIBE, 1979, p. 92)

A este nivel estuvo primero únicamente Estados Unidos desde el fin de la Segunda Guerra Mundial hasta los años sesenta, cuando se produjo la paridad estratégica.

La segunda categoría:

“(...) se caracteriza por la inexpugnabilidad del territorio propio, combinada con el ejercicio de una hegemonía sobre determinadas áreas y una presencia preponderante en otras, aunque también restringidas a ciertas aéreas (...).” (JAGUARIBE, 1979, p. 92)

Un ejemplo de ello fue la URSS hasta su asenso a la categoría superior y el caso de la China de Mao tras la ruptura con los soviéticos en los años sesenta.

En tercer lugar aparecen la autonomía, la cual: “(...) sin asegurar la inexpugnabilidad del territorio propio, se caracteriza por el hecho de que los titulares disponen de medios para imponer severas penalidades, materiales y morales, a un eventual agresor."(JAGUARIBE, 1979, p. 93)

Además tiene: “(...) un margen bastante amplio de autodeterminación en la condición de sus negocios internos y de una apreciable capacidad de actuación internacional independiente (...).” (JAGUARIBE, 1979, p. 93)

El último nivel es el de dependencia que:

(...) incluye a la gran mayoría de los países del mundo. En él se encuentran los países que no disponen de requisitos para ubicarse en niveles más altos. Tales países, a excepción de algunos remanentes coloniales, poseen nominalmente la condición de Estados soberanos, dotados de órganos propios de gobierno y acreditados como interlocutores independientes ante otros Estados y Organismos internacionales. (JAGUARIBE, 1979, p. 93)

Uno de los rasgos salientes de estos niveles es que "no son estables y permanentes", sino absolutamente dinámicos. (JAGUARIBE, 1979, p. 96)

Particularmente en el caso de los países latinoamericanos existen además tres elementos básicos para conseguir un estado más autónomo (JAGUARIBE, 1974, p. 106):

I. el desarrollo global de las sociedades nacionales;

II. el desarrollo debe ir acompañado de la generación de margen de maniobra que mejore la capacidad de toma de decisiones y un mayor control sobre los actores nacio- 
nales a sí mismos, y la capacidad de generar el exterior para reducir la dependencia de la posición de los países desarrollados en general y de los EE.UU. en particular;

III. tanto el desarrollo y la autonomía se van a desarrollar en las condiciones más favorables para su "autorealización”.

Jaguaribe anota que la principal permisividad internacional para estos países se da cuando "sus necesidades de capital chocan con su necesidad de afirmación y consolidación nacional” (JAGUARIBE, 1973, p. 54).

Bologna rescata esa estrategia común pues:

(...) tiende al aumento de la capacidad negociadora del Sur frente al Norte, es superadora del atraso y del estancamiento individual de los países del área y logrará una estructuración de la sociedad internacional donde los paradigmas de la misma, sean la justicia social y la solidaridad entre todos los actores que intervienen en el escenario internacional. (BOLOGNA, 1989, p. 258)

Por ese motivo, para Puig:

Helio Jaguaribe es uno de los teóricos que ha impugnado con tenacidad por demostrar empírica y teoréticamente, global y coyunturalmente, las posibilidades concretas de autonomización para determinados Estados periféricos... (PUIG, 1984, p. 18)

Para Bologna entre ambas perspectivas, a pesar de sus semejanzas:

(...) es posible realizar una distinción analítica entre el enfoque de la autonomía de Puig y el de la viabilidad de Jaguaribe. El aporte de Puig se centra en el estilo o práctica autonomista que ven aportar los países de América Latina en cambio, el aporte de Jaguaribe pone el énfasis en las condiciones del accionar internacional. (BOLOGNA, 1987, p. 23)

Además de esta diferencia Puig entiende que es necesario resaltar el rol de las elites, cuando éstas estén “decididas a emprender el camino de la autonomización” (PUIG, 1980, p. 154).

\subsection{AUTONOMÍA PUGIANA}

Juan Carlos Puig observó que eran necesarios análisis que apuntaran a comprender "estructuralmente mediante la selección de variables relevantes y significativas"(3) y que permitan "por lo menos delinear las tendencias relevantes profundas y apreciar los errores y aciertos en función del logro de una mayor autonomía para el país” (PUIG, 1984, p. 91).

Para el autor en el sistema internacional existen condiciones asimétricas y un complejo (e inestable) de equilibrio de poder, creado por una división de funciones: Repar-

(3) Esta idea de fuerza profunda, o tendencia, como la llamó Puig, fue tomada tanto por éste como por Jaguaribe de las obras de Renouvin y Duroselle, quienes plantean: "las iniciativas de los estadistas quedan determinadas en gran medida por fuerzas profundas, es decir, la influencia de las masas." Así, este accionar de los gobiernos se explicaba tanto por las condiciones económicas como de los sentimientos y pasiones colectivas. (Duroselle, 1991: 128) 
tidores Supremos (toman las decisiones macro y vigilan su cumplimiento), Repartidores Inferiores (son los gobiernos que siguen las decisiones de macro, pero con algunos márgenes de maniobra y ajustes de ellas) y, los Recipientários (el resto de la población del planeta) (PUIG, 1980, p. 145).

El régimen internacional se articulaba según los siguientes criterios: la posesión de armas de destrucción masiva, la impermeabilidad interbloque, la autonomización intrabloque, la ruptura estratégica, la permeabilidad extrabloque y la prohibición del uso de la fuerza para las potencias medianas y pequeñas.

La posesión de armas de destrucción masiva es el criterio que ordena el sistema internacional. Las naciones que tienen armas nucleares están en el centro de las decisiones. La Impermeabilidad Interbloquees el acuerdo entre las superpotencias que todo lo que ocurre dentro de su área no se verá influido por el otro. La autonomización es el aumento de la "libertad de acción" por parte de los socios menores de la coalición, en donde los actores nacionales ajustan sus intenciones a una ecuación entre el nivel actual de la autonomía y de su alcance potencial. La ruptura estratégica es cuando el estado periférico decide cortar lazos con gran poder. La permeabilidad extrabloque es comprender el proceso de descolonización y el surgimiento de nuevos estados y la disputa entre las superpotencias por su dominio. La prohibición del uso de la fuerza para potencias medianas y pequeñas depende de los criterios del bloque y el interés de las superpotencias. Si está de acuerdo, está autorizado. Si no, está prohibido (PUIG, 1984, pp. 39-110).

Además de estos criterios en Puig se establecen categorías (PUIG, 1984, pp. 74-79) de dependencia y autonomía:

- Dependencia paracolonial: las élites que conducen ese estado periférico se consideran un apéndice político, económico e ideológico de la metrópoli;

- Dependencia racionalizada: aquí las elites tienen un proyecto nacional pero dependiente del centro;

- Autonomía heterodoxa: aquí la elite autonomista no cruza los intereses estratégicos de la Gran Potencia, pero sus expectativas y proyectos pueden, o no, coincidir con los deseos de la metrópoli; y que en su accionar separa cuando están en juego los intereses de la Metrópoli o del Bloque;

- Autonomía Secesionista: es la ruptura con la potencia hegemónica. No es recomendable, ya que implica una superación de las capacidades nacionales, causando problemas de política interior y exterior.

Como vemos el rol de las elites resultan fundamentales, pero como ha notado Raúl Bernal Meza, el pasaje de "la "dependencia" a la "autonomía" podría producirse solo en la medida que los países avancen en materia de viabilidad nacional” (MEZA, 2013).

Este concepto desarrollado por Jaguaribe resulta un elemento articulador de ambas concepciones, además de que son dinámicos y no estáticos.

Mario Rapoport critica el concepto de autonomía heterodoxa por considerarlo rígido y esquemático, aunque lo considera un concepto "sugerente" para abrir un espacio de pensamiento y acción desde la periferia (RAPOPORT, 1990, p. 565). 


\subsection{LA AUTONOMÍA EN TOMASSINI}

Es el cuarto aporte a la teoría de la autonomía, este autor chileno que comenzó su carrera en la CEPAL, y la continuó en la Universidad de Chile, representa a una generación distinta a la de Jaguaribe y Puig por eso presentó una lectura internacional más en consonancia al clima de los años ochenta de crisis de los "grandes relatos" y transformaciones en el sistema global (KLAVEREN, 2010, p. 143).

Fue uno de los promotores del Proyecto RIAL que buscaba la promoción y el perfeccionamiento de los estudios de Relaciones Internacionales en América Latina; mantenerlos desde una perspectiva latinoamericana, y fomentar un enfoque multidisciplinario de Relaciones Internacionales que se alejen de los estudios más tradicionales alrededor de la historia diplomática y la geopolítica (TOMASSINI, 1980, p. 546).

El autor propone (1980, p. 556) algunos elementos para pensar el sistema internacional:

I. las Relaciones Internacionales no deberían centrarse sólo en el poder;

II. pensarlas en términos teóricos y abstractos;

III. remediar el segundo debate;

IV. el sistema internacional no está compuesto únicamente por estados;

V. ampliar la agenda de investigación.

Si bien como hemos expuesto muchos de estos presupuestos fueron abordados por Jaguaribe y Puig, creemos que este proceso obedece a una crisis paradigmática que en el caso argentino ubicamos a partir de 1983 y donde la nueva generación de autores busca legitimar su posición frente a aquellos.

El punto donde si hay una marcada diferencia entre el autor chileno y sus pares argentino y brasileño es la crítica al concepto del Tercer Mundo. Para el (TOMASSINI, 1977, p. 113), la idea del Tercer Mundo se basa en falacias ya que representa una pluralidad que es difícil conceptualmente: micos;

I. hay elites nacionales, pero varían mucho en sus aspectos ideológicos y econó-

II. los problemas sólo pueden resolverse multilateralmente, con la participación del Tercer Mundo;

III. como se dijo anteriormente, el Tercer Mundo no es homogéneo y no puede ser tratado como tal.

Otro aspecto diferencial es que para Luciano Tomassini la autonomía es un fenómeno producto de la concurrencia de dos factores, la apertura y el grado de intervención estatal. Entendiendo a la primera como los modos y momentos de la participación del Estado en las iniciativas internacionales para lograr sus objetivos nacionales. Y el segundo es la capacidad de establecer una estrategia y ser capaz de ejecutarlo. 

188):

A partir del cruce de éstos encuentra cuatro modelos (TOMASSINI, 1982, pp. 187-

I. Apertura con la intervención (p. e. Brasil y Corea del Sur);

II. Apertura sin la intervención (p. e., Chile);

III. de aislamiento con Intervención (Argentina de los cincuenta a los ochenta);

IV. aislamiento sin intervención (Cuba y Corea del Norte).

La idea de Tomassini reconoce que la periferia está subordinada al capitalismo central, pero reconoce que es necesario:

(...) una reinterpretación de las relaciones entre los dos segmentos del sistema, a la luz de la cual probablemente los centros presenten una imagen menos monolítica y algunos países en desarrollo ocupen una posición menos periférica. (TOMASSINI, 1982, p. 174)

Esta dinámica de una fragmentación mayor de la periferia, sumada a una interpretación de la existencia de nuevos desafíos en materia de seguridad y económicos, se reflejan en sus escritos que acompañan los cambios de pensamiento latinoamericano en los años ochenta y noventa, y de los cuales el proyecto del RIAL fue un instrumento.

Y además son un reflejo en materia internacional de las variaciones de la CEPAL, ante el abandono de la reestructuración económica a favor del crecimiento hacia los términos de competitividad internacional en el libre mercado en un sentido neoliberal.

\section{CONCLUSIONES}

Las teorías de la autonomías aparecen en las décadas que van desde los sesenta hasta los ochenta en el Cono Sur de América, producto de los debates internacionales y latinoamericanos sobre la situación internacional de la región.

Pero en esos elementos de unidad espacial y temporal producen también los elementos que preanuncian un amplio abanico de opciones. La relación de la autonomía con el realismo, más como una cuestión filosófica que metodológica, en donde la impronta del Segundo Debate resulta central.

La determinación de variables internas a la hora de pensar la Autonomía es un elemento nacido de aquella discusión y que separa nuestras visiones del realismo clásico de la inmediata posguerra.

El pensamiento cepalino por un lado, aportó un elemento nodal, la división del mundo entre Centro y Periferia que determina nuestros comportamientos de acuerdo en qué lado estemos, aunque sus aportes no terminan allí, elementos como la lucha contra el intercambio desigual, la integración y la promoción de la industrialización son centrales para la realización del proyecto autonómico.

Aunque existen diferencias, ya que mientras la teoría cepalina ve a la autonomía como una de las bases para generar un modelo de inserción de la periferia en el esquema 
capitalista global con competitividad y desarrollo social, en el caso de algunas versiones de la Teoría de la Dependencia es un medio para la ruptura radical, ya sea promovida por el Estado o por los movimientos sociales.

Esta apertura marca el campo en los cuales se desarrolla la teoría autonómica, en la cual Jaguaribe tiene varios puntos de encuentro con Puig, en donde la versión periférica del primero y la heterodoxa del segundo son muy similares, o la confluencia en el criterio de viabilidad.

Pero se diferencian por las preocupaciones de cada uno, mientras el brasileño la cohesión interna estaba dada por el desarrollo de las sociedades, para Puig la puja política entre la elite dependentista y la autonómica resultan centrales en sus análisis.

A pesar de utilizar criterios para describir los regímenes internacionales en el marco de la Guerra Fría, lo cierto es que la cuestión de la asimetría entre los poderosos del sistema y los países candidatos a la autonomía ocupan el eje de sus análisis, por lo tanto se puede aplicar escenarios unipolares o multipolares.

La aparición de una forma de autonomía a principios de los ochenta, marca el cierre del ciclo inicial de las innovaciones teóricas de las relaciones internacionales conosureñas, es también el punto de inicio de otra historia.

\section{BIBLIOGRAFÍA}

ARON, Raymond. Pazy Guerra entre las Naciones. Madrid: Alianza Editorial, 1962.

BOLOGNA, Alfredo Bruno. Teorías y propuestas de relaciones internacionales para los países del Sur. Rosario: CERIR, 1987.

BIELSCHOWSKY, Ricardo. Diversificación productiva y exportadora: las cuatro primeras décadas. In: BIELSCHOWSKY, Ricardo; IZAM, Miguel; MULDER, Nanno. Dos estudios de la evolución del pensamiento de la CEPAL sobre la diversificación productiva y la inserción internacional (1949-2008). Santiago de Chile: Ediciones Naciones Unidas, 2012.

BOLOGNA, Alfredo Bruno. Los aportes de Juan Carlos Puig a las Relaciones Internacionales. Mundo Nuevo. Revista de estudios latinoamericanos, Caracas, v. 07, n. 44-46, pp. 251-258, 1989.

CARDOSO, Fernando Henrique. A soma e o resto. Um olhar sobre a vida aos 80 anos. São Paulo: Civilização Brasileira, 2011.

CORIGLIANO, Francisco. Veinte años no es nada: un balance de los debates teóricos acerca de la política exterior argentina. Friedrich Ebert Stiftung, 2009. Disponible en: <http://www.seguridad-regional-fes.org/upload/3699-001_g.pdf>. Acesso em: 18.3.2014.

DUROSELLE, Jean Baptiste. Todo imperio perecerá. México: Fondo de Cultura Económica, 1991.

IÑIGUEZ, Carlos Piñeiro. Herejías Periféricas. Raúl Prebisch. Vigencia de su pensamiento. Buenos Aires: Grupo Editor Latinoamericano, 2003.

JAGUARIBE, Helio. Desarrollo económico y político. México: Fondo de Cultura Económica, 1973. JAGUARIBE, Helio. Brasil: crisis y alternativas. Buenos Aires: Amorrortu, 1974.

JAGUARIBE, Helio. Autonomía periférica y hegemonía céntrica. Estudios Internacionales. Santiago de Chile, n. 49, p. 91-130, 1979. 
KAY, Cristóbal. Estructuralismo y teoría de la dependencia en el periodo neoliberal: Una perspectiva latinoamericana. Nueva Sociedad, Caracas, n. 158, p. 100-119, 1998.

KLAVEREN, Alberto Van. El análisis de la política exterior latinoamericana: perspectivas teóricas. In: MUÑOZ, Heraldo; TULCHIN, Joseph. Entre la autonomía y la subordinación. Política exterior de los países latinoamericanos. Buenos Aires: Grupo Editor Latinoamericano, 1984.

KLAVEREN, Alberto Van. Luciano Tomassini: académico y artífice 1935-2010.Estudios Internacionales, Santiago, v. 43, n. 166, p. 143-147, 2010.

KONART, Gerhald Drekonia. Aproximaciones a la política exterior Latinoamericana. Estudios internacionales, Santiago de Chile, v. 14, n. 53, p. 89-104, 1981.

MEZA, Raúl Bernal. Los caminos de la autonomía desde la lectura de la obra de Juan Carlos Puig. In: Mundo Nuevo. Revista de estudios latinoamericanos, Caracas, v. 07, n. 44-46, p. 236-240, 1989.

MEZA, Raúl Bernal. Heterodox Autonomy Doctrine: realism and purposes and its relevance. Revista Brasileña de Política Internacional, Brasília, v. 56, n. 02, p. 45-62, 2013.

NOVOA, Carlos Torres. A teoría de la dependencia: nota crítica sobre su metodología histórico-estructural. Nueva sociedad, Caracas, n. 42, p. 70-86, 1979.

PUIG, Juan Carlos. Doctrinas internacionales y Autonomía latinoamericana. Caracas: Universidad Simón Bolívar/Instituto de Altos Estudios de América Latina, 1980.

PUIG, Juan Carlos. América Latina: políticas exteriores comparadas. Buenos Aires: Grupo Editor Latinoamericano, 1984.

O’DONNELL, Guillermo y Linck, Delfina. Dependencia y autonomía. Formas de dependencia y estrategias de liberación. Buenos Aires, Amorrurtu, 1973.

PEDRAZA, Luis Dallanegra. Realismo-Sistémico-Estructural: La Política Exterior como "Construcción" de Poder. Córdoba: Edición del Autor, 2009

PRADO, Fernando Correa. Otras razones del neodesarrollismos (o porqué se desconoció a la teoría marxista de la dependencia). Argumentos, México, v. 26, n. 72, p. 99-126, 2013.

RAPOPORT, Mario. Problemas y etapas en la historia de las relaciones internacionales de la Argentina In Historiografía Argentina (1958-1988). Un evaluación crítica de la producción histórica argentina. Buenos Aires: Comité Internacional de Ciencias Históricas, 1990.

RASCOVAN, Alejandro. Teorías críticas en Relaciones Internacionales; Marx(ismo); imperialismos; teoría de la dependencia. In: LLENDERROZAS, Elsa. Relaciones Internacionales: teorías y debates. Buenos Aires: Eudeba, 2013.

SANTOS, Theotonio dos. La teoría de la dependencia. Balance y perspectivas. Buenos Aires: Plaza \& Janés, 2003.

SIMONOFF, Alejandro. Teorías en movimiento. Los orígenes disciplinares de la política exterior y sus interpretaciones históricas. Rosario: Prohistoria Ediciones, 2012.

SUNKEL, Osvaldo. Capitalismo transnacional y desintegración nacional en América Latina. Buenos Aires: Nueva Visión, 1972

TICKNER, Arlene. Los estudios internacionales en América Latina. ¿Subordinación intelectual o pensamiento emancipatorio? Bogotá: Alfaomega/Uniandes, 2002.

TOMASSINI, Luciano. Falencias y falacias: notas sobre el estudio de las relaciones Norte-Sur. Estudios internacionales, Santiago de Chile, v.10, n. 40, p.111-130, 1977.

TOMASSINI, Luciano. Los Estudios Internacionales en América Latina: algunas contribuciones. Estudios Internacionales, Santiago de Chile, v. 13, n. 52, p. 310-326, 1980.

TOMASSINI, Luciano. Interdependencia y desarrollo nacional.Estudios Internacionales, Santiago de Chile, v. 15, n. 58, p. 166-189, 1982. 